TITLE:

\title{
Model-based registration for pneumothorax deformation analysis using intraoperative cone- beam CT images
}

\section{$\operatorname{AUTHOR}(S):$}

Maekawa, Hinako; Nakao, Megumi; Mineura, Katsutaka; Chen-Yoshikawa, Toyofumi F.; Matsuda, Tetsuya

\section{CITATION:}

Maekawa, Hinako ... [et al]. Model-based registration for pneumothorax deformation analysis using intraoperative conebeam CT images. 2020 42nd Annual International Conference of the IEEE Engineering in Medicine \& Biology Society (EMBC) 2020: 5818-5821: 9176729.

\section{ISSUE DATE:}

2020

URL:

http://hdl.handle.net/2433/265388

\section{RIGHT:}

(C) 2020 IEEE. Personal use of this material is permitted. Permission from IEEE must be obtained for all other uses, in any current or future media, including reprinting/republishing this material for advertising or promotional purposes,

creating new collective works, for resale or redistribution to servers or lists, or reuse of any copyrighted component of this work in other works.; This is not the published version. Please cite only the published version. この論文は出版社版

でありません。引用の際には出版社版をご確認ご利用ください。 


\title{
Model-based registration for pneumothorax deformation analysis using intraoperative cone-beam CT images
}

\author{
Hinako Maekawa ${ }^{1}$, Studient Member, IEEE, Megumi Nakao ${ }^{1}$, Member, IEEE, Katsutaka Mineura ${ }^{2}$, \\ Toyofumi F. Chen-Yoshikawa ${ }^{3}$, and Tetsuya Matsuda ${ }^{1}$, Member, IEEE
}

\begin{abstract}
Because the lung deforms during surgery because of pneumothorax, it is important to be able to track the location of a tumor. Deformation of the whole lung can be estimated using intraoperative cone-beam CT (CBCT) images. In this study, we used deformable mesh registration methods for paired CBCT images in the inflated and deflated states, and analyzed their deformation. We proposed a deformable mesh registration framework for deformations of partial organ shapes involving large deformation and rotation. Experimental results showed that the proposed methods reduced errors in point-to-point correspondence. As a result of registration using surgical clips placed on the lung surface during imaging, it was confirmed that an average error of $3.9 \mathrm{~mm}$ occurred in eight cases. The result of analysis showed that both tissue rotation and contraction had large effects on displacement.
\end{abstract}

\section{INTRODUCTION}

Three-dimensional (3D) high-resolution computed tomography (CT) images and magnetic resonance imaging (MRI) are widely used to analyze organ deformation and shape differences. Intraoperative pneumothorax can involve large deformations of the lung contour and changes in position of any target lesions in the parenchyma. Some studies have simulated organ deformation and estimated the tumor position for intraoperative image guidance[1][2]. If pneumothorax deformation can be mathematically modeled, based on image analysis, the position of a tumor during surgery can be estimated more accurately.

Different registration techniques have been studied to obtain local correspondence between organ shapes in medical images. Examples of studies that performed registration for organ deformation include the investigations that performed preoperative CT and intraoperative CT registration for the liver and lung[3][4] and a study that performed registration during the respiratory cycle[5]. 3D analyses of pneumothorax deformation of animal lungs have been conducted[6][7]. In recent years, it has become possible to partially image organs using intraoperative cone-beam CT (CBCT), which has been used to estimate the changes between preoperative CT and intraoperative CBCT. Studies on image registration for animal lungs[8], abdominal image registration[9], and analysis of preoperative CT and intraoperative CBCT images in the inflated state[10] have been reported. There have been

${ }^{1}$ H. Maekawa, M. Nakao, and T. Matsuda are with the Department of System Science, Graduate School of Informatics, Kyoto University, Kyoto, 606-8501, Japan (e-mail: mhina@ sys.i.kyoto-u.ac.jp)

${ }^{2} \mathrm{~K}$. Mineura is with the Department of Thoracic Surgery, Kyoto University Hospital, Kyoto, 606-8507, Japan

${ }^{3}$ T. F. Chen-Yoshikawa is with the Department of Thoracic Surgery, Nagoya University Hospital, Nagoya, 466-8550, Japan

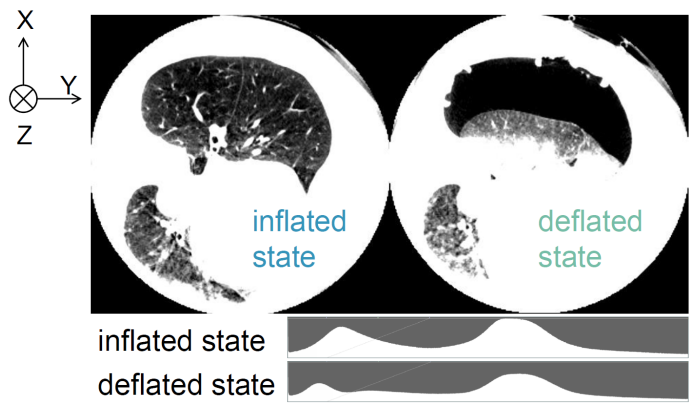

Fig. 1. Paired CBCT images(window width: 454, window level: -628) and histogram of $\mathrm{CT}$ values

no reports of intraoperative pneumothorax in humans, and inter-patient variations of deformation have not been studied because of difficulties with intraoperative measurement.

This study was performed to register and analyze the shape of the part of the lung included in the CBCT images. We designed a mesh registration framework for shape changes involving extreme deformation with rotation. The registered meshes with local correspondences are directly available for surgical guidance in thoracoscopic surgery, and for building statistical deformation models with inter-patient variations.

This paper reports the findings of pneumothorax deformation analysis with the registration of CBCT images of inflated and deflated states.

\section{METHOD}

\section{A. Data}

In this study, two images, one of inflated and one of deflated states were generated from lung cancer patients at Kyoto University Hospital. The images are measured in the same position in the inflated and deflated states by controlling the bronchial pressure after thoracotomy.

Each CBCT volume consists of a set of sections of 512 $\times 512$ pixels (pixel resolution: $0.49 \mathrm{~mm} \times 0.49 \mathrm{~mm} \times 0.49$ $1.0 \mathrm{~mm})$. In this paper, this dataset is referred to as "paired CBCT images", and the example is shown in Fig. 1. Air flows around the lung and it collapses. CT density readings of lung parenchyma differ depending on the amount of air in the lungs. As image-based registration did not work properly, we applied deformable mesh registration (DMR) to the meshes of lung surfaces extracted from CBCT images.

The images are measured with two surgical clips placed on the lung surface near the tumor. They can be used as 


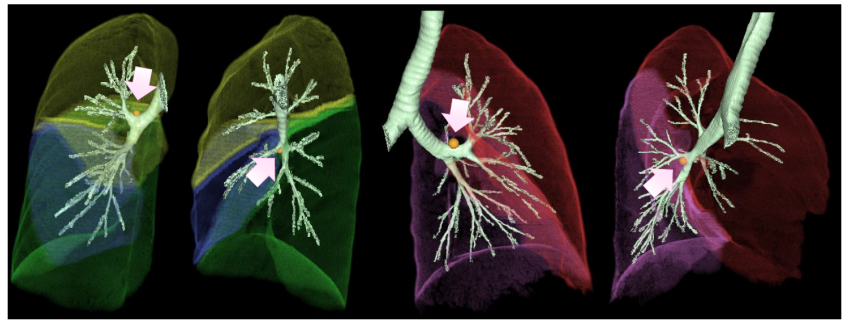

(a)

(b)

(c)

(d)

Fig. 2. Reference points set by the respiratory surgeon. (a) reference point1(right lung), (b) reference point2(right lung), (c) reference point3(left lung), (d) reference point 4 (left lung)

landmarks. Besides, two reference points were manually set by the surgeon near the first bronchial branch on the boundary of the upper and lower lobes(Fig. 2).

3D labeling of lungs, bronchi, and tumors was performed from CBCT images using Fujifilm Synapse VINCENT (Fujifilm Corporation, Tokyo, Japan). Surface meshes of lungs with 500 vertices and 996 triangular elements were generated. The number of vertices was determined by balancing the need for accuracy against the calculation time. In this study, the whole lung was treated as one shape because segmentation of the lung lobes is difficult because of limitations of image quality or existence of artifacts in CBCT images.

There are some differences in the patient's posture and air contents of the lung. Therefore, the normalization of the lung mesh is first performed. First, the translation is performed so that the midpoint between the two reference points is the origin. The individual posture is normalized by matching the axis connecting the two reference points to the inflated state.

\section{B. Problem definition}

In this study, we focused on the following two issues for DMR of intraoperative pneumothorax deformation;

\section{Partial lung shapes in CBCT images \\ 2 Large deformation with rotation}

First, the entire lung cannot be measured because of the limited imaging area in CBCT imaging. As shown in Fig. $3(\mathrm{a})$, the imaging area for the lung may change between inflated and deflated lungs; some parts of the lung in the deflated state may be located outside of the imaging area in the inflated state. Here, the reference points and clips can be used as landmarks. Based on the location of the landmarks, we can consider the following three types of partial shapes measured in CBCT images;

TYPE 1 Top of lung

TYPE 2 Center of lung

TYPE 3 Bottom of lung

Fig. 4(a) shows the region of interest (ROI) defined for the three types of partial shapes. The areas among the reference points and clips are considered to be the corresponding ROIs of the inflated/deflated lungs. For TYPE 1, the lower lobe side area outside these points is excluded, and for TYPE 3, the upper lobe side area outside these points is excluded.

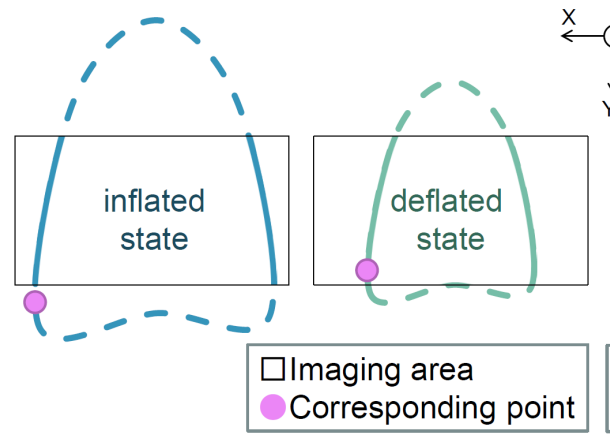

(a)

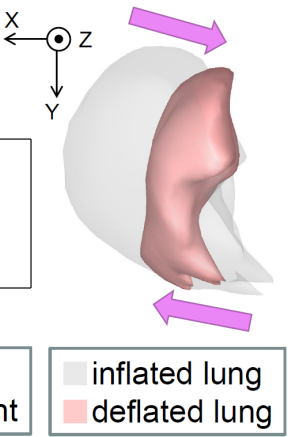

(b)
Fig. 3. Problem definition.(a) corresponding points in the inflated state are located outside of the imaging area, (b) an example of paired CBCT with large deformation and rotation

Since TYPE 2 has a corresponding point only in the middle area among these points, the upper and lower lobe side areas outside these points are excluded. The boundary surface is parallel to the boundary surface of the CBCT partial shape. Fig. 4(b) shows vertices used for registration.

For the second point of the problem, registration errors tend to increase in case of large deformations with rotation. Fig. 3(b) shows an example of the paired CBCT. In the case of pneumothorax deformation, some cases largely rotate in this way, so in this study, clips were used to estimate the rotation.

\section{DMR for pneumothorax deformation}

In this section, the registration algorithm for obtaining local correspondence between paired CBCT is explained. As described in Section II-B, registration errors tend to increase in cases of extreme deformation with rotation, so this study use reference points and clips. Shape matching is performed from the source shape to the target shape. In this study, inflated states were used as the source and deflated states were used as the targets. Registration is performed in the next step.

STEP 1 Affine Transformation

STEP 2 Piecewise Affine Transformation[11]

STEP 3 Laplacian-based Shape Matching[7]

First, in STEP 1, the rotation and scale are adjusted, and then, in STEP 2, Piecewise Affine Transform (PWA), which performs Affine Transformation on each of the divided regions, is employed. Here, the source is updated to minimize the objective function defined by Eq. (1).

$$
\begin{aligned}
E & =E_{\text {shape }}+E_{\text {clip }} \\
& =d\left(S^{D}, S^{I}\right)+w\left|\boldsymbol{p}^{D}-\boldsymbol{p}^{I}\right|
\end{aligned}
$$

where $d$ is the mean distance[7], mean value of the nearest bidirectional point-to-surface distance of the two meshes $S^{D}$ and $S^{I} . w$ is a weight, and $\boldsymbol{p}^{D}$ and $\boldsymbol{p}^{I}$ are the position of surgical clips placed on the lung surface in the inflated and deflated states, respectively. In STEP 1, the evaluation function based on the clip and the reference $E_{c l i p}$ is minimized, and in STEP 2, $E$ is minimized in consideration of 


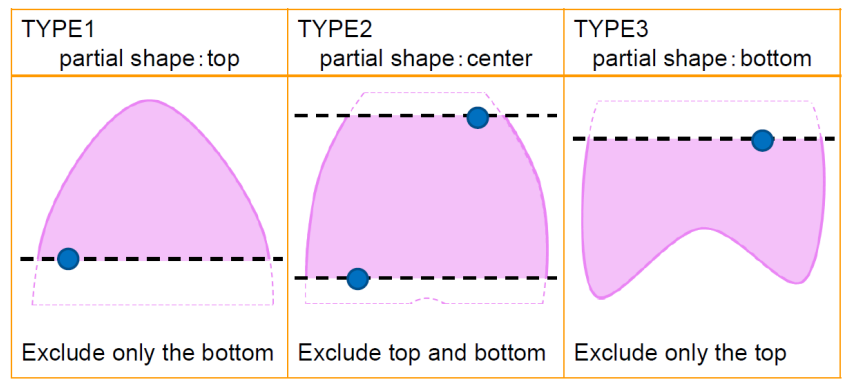

(a)

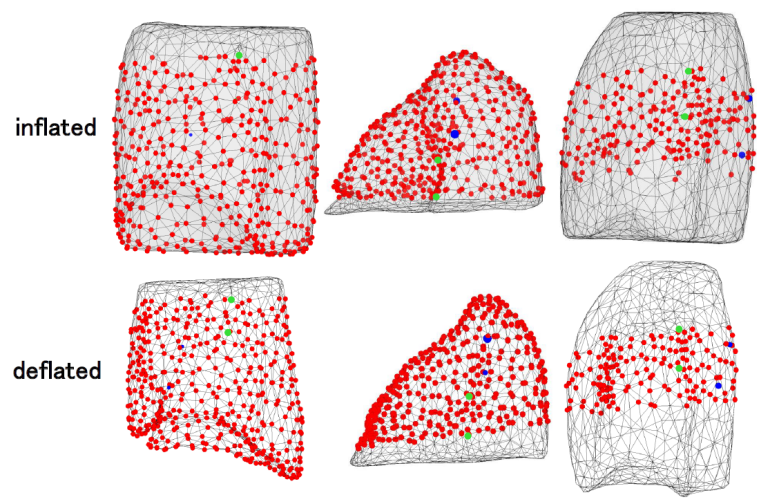

(b)

Fig. 4. Processing of ROI. (a) The processing method of ROI by type, (b) Red: corresponding ROIs of the mesh, green: reference points, blue: clips

the shape evaluation function $E_{\text {shape }}$. Here, to calculate $d$, the nearest two vertices are determined as the pair of vertices that minimize the local shape similarity $Q$ defined by Eq. (2).

$$
Q=\left|\boldsymbol{v}_{s}-\boldsymbol{v}_{t}\right|+\gamma\left(1-\boldsymbol{n}_{s} \cdot \boldsymbol{n}_{t}\right)
$$

$\boldsymbol{v}_{s}$ is the source vertex, $\boldsymbol{v}_{t}$ is the target vertex, $\boldsymbol{n}_{s}$ is the source normal vector, $\boldsymbol{n}_{t}$ is the target normal vector, and $\gamma$ is a weight. $Q$ locally evaluates shape similarity per vertex, and the pair of vertices that minimize $Q$ is set as the nearest vertices. Then, $d$ is computed as the mean bidirectional pointto-surface distance defined by the vertex pair.

Finally, in STEP 3, registration is performed to match the surfaces with high accuracy. The discrete Laplacian is a shape descriptor, and by using it, minimizes shape differences while maintaining shape. Regarding the details of the algorithms and the performance, see [7].

\section{Analysis method}

This section describes how we analyzed pneumothorax deformation. Investigating the characteristics of the rotation of the pneumothorax can lead to better registration. Therefore, the displacement of clips is decomposed into a contraction component, which is the displacement in the direction of the origin, and a rotation component, which is obtained by removing the contraction component from the displacement. Assuming that pneumothorax deformation does not move the hilum, we set it as the origin. For vertices, displacement can be represented by scaling and rotation around the origin.

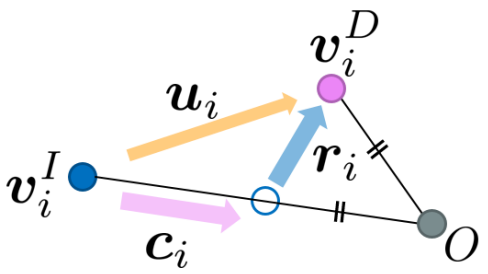

Fig. 5. Relationship between total displacement $\boldsymbol{u}_{i}$, contraction component $\boldsymbol{c}_{i}$, rotation component $\boldsymbol{r}_{i}$ computed from the corresponding vertices $\boldsymbol{v}_{i}^{I}$ and $\boldsymbol{v}_{i}^{D}$.

Fig. 5 shows the relationship between displacement and each component. The displacement $\boldsymbol{u}_{i}$, the contraction component $\boldsymbol{c}_{i}$, and the rotation component $\boldsymbol{r}_{i}$ are expressed by the Eq. (3), (4), (5).

$$
\begin{array}{r}
\boldsymbol{u}_{i}=\boldsymbol{v}_{i}^{D}-\boldsymbol{v}_{i}^{I} \\
\boldsymbol{c}_{i}=\left(\left|\boldsymbol{v}_{i}^{D}\right|-\left|\boldsymbol{v}_{i}^{I}\right|\right) \frac{\boldsymbol{v}_{i}^{I}}{\left|\boldsymbol{v}_{i}^{I}\right|} \\
\boldsymbol{r}_{i}=\boldsymbol{u}_{i}-\boldsymbol{c}_{i}
\end{array}
$$

The distance between clips and the origin is used as an index of the position of clips.

\section{EXPERIMENTS AND RESULTS}

We used data as described in section II-A. Cases 1-5 are the right lung and cases 6-8 are the left lung, for a total of 8 cases. We compared two registration methods with/without surgical clips for the constraints. To perform analysis based on the correct displacement, 10 registrations were made in each case and the one with the least clip position error was used. Regarding the weight parameters, we used 10.0 for $\delta$ and 1.0 for $\lambda$ after the examination of several parameters sets.

\section{A. Registration results}

Comparing the registrations that use and do not use clips. Table I shows the registration errors of the two methods. Mean distance (MD), Hausdorff distance (HD)[7] and target registration errors (TRE) of the two surgical clips are used for the evaluation criteria. MD and HD were almost equal. TRE1 and TRE2 were $20.4 \mathrm{~mm}$ and $21.4 \mathrm{~mm}$ without clips but were $3.9 \mathrm{~mm}$ and $4.0 \mathrm{~mm}$ with clips. If clips were not used, the surface could be registered with high accuracy, but the position of the clips was not very accurate. Registration was more accurate when using clips.

Without clips, if the organ is rotating as shown in Fig. 3(b), the algorithm simply locates nearby vertices and cannot find the corresponding vertex. By using clips as landmarks, the effects of rotation, which are difficult to find only by shape, can be ascertained.

From this result, it was found that the conventional method cannot cope with the rotation, and the error increases. Our method requires landmarks and cannot be used in clinical settings. Therefore, a registration method that supports rotation will be required. 
TABLE I

QUANTITATIVE COMPARISON OF MEAN (MINIMUM - MAXIMUM) REGISTRATION ERRORS. MEAN DisTANCE (MD), HAUSDORFF DistancE (HD) AND TWO TARGET REGISTRATION ERRORS (TRE) ARE LISTED.

\begin{tabular}{ccc}
\hline & no clips & with clips \\
\hline MD [mm] & $0.1(0.1-0.2)$ & $0.1(0.1-0.2)$ \\
HD [mm] & $1.8(0.8-4.6)$ & $1.5(0.7-3.2)$ \\
TRE1 $[\mathrm{mm}]$ & $20.4(7.5-39.3)$ & $3.9(1.8-6.8)$ \\
TRE2 $[\mathrm{mm}]$ & $21.4(7.9-35.8)$ & $4.0(2.2-5.8)$ \\
\hline
\end{tabular}
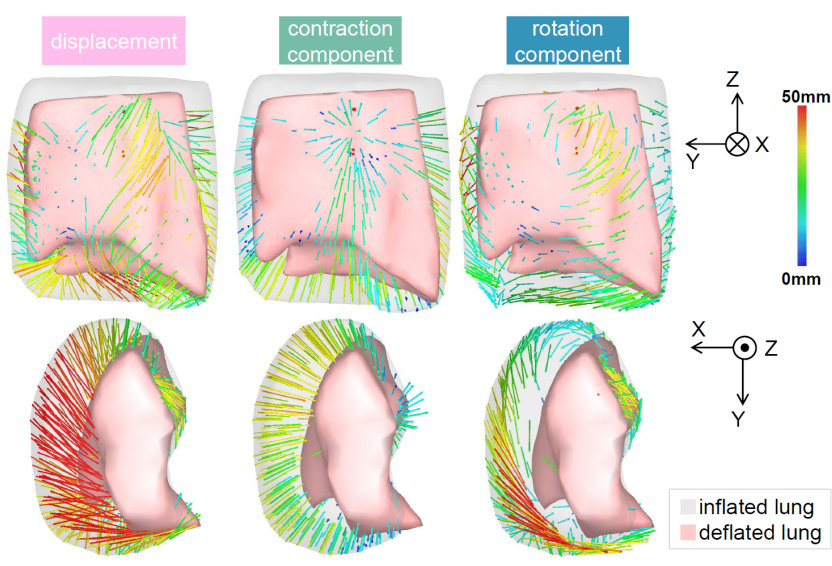

Fig. 6. Visualization results of total displacement, contraction and rotation components.

\section{B. Analysis of pneumothorax deformation}

Fig. 6 shows, from left to right, the results of total displacement, contraction, and rotation components. The displacement was decomposed into a contraction and a rotation component, and both components had large vectors. The contraction component tended to have a large vector in the direction of gravity. There was a part with a rotation component of $50 \mathrm{~mm}$ or more (red vector). Table II shows the average values of the displacement, contraction, and rotation components. The smallest rotation component was $5.8 \mathrm{~mm}$ and the largest one was $36.7 \mathrm{~mm}$. Also, the contraction component was a minimum of $8.1 \mathrm{~mm}$ and a maximum of $31.4 \mathrm{~mm}$, and there is a difference between cases.

Differences in rotation and contraction were observed within and between cases. It is thought that friction and adhesions between the lobes and surrounding organs made this difference.

\section{CONCLUSIONS}

In this study, registration and analysis were performed with clips using CBCT images, which are partially observed data containing large rotations. In future work, we will investigate the relationship between lung shape and deformation and develop a registration method that supports rotation.

\section{ACKNOWLEDGMENT}

This research was supported by the Japan Agency for Medical Research (AMED) and the Acceleration Transformative Research for Medical Innovation (ACT-M) Program.
TABLE II

THE AVERAGE VALUES OF DISPLACEMENT, CONTRACTION AND ROTATION COMPONENT.

\begin{tabular}{cccc}
\hline cases & $\begin{array}{c}\text { Displacement } \\
{[\mathrm{mm}]}\end{array}$ & $\begin{array}{c}\text { Contraction } \\
{[\mathrm{mm}]}\end{array}$ & $\begin{array}{c}\text { Rotation } \\
{[\mathrm{mm}]}\end{array}$ \\
\hline case1 & 43.6 & 31.4 & 24.8 \\
case2 & 11.9 & 8.1 & 8.0 \\
case3 & 21.9 & 20.4 & 5.8 \\
case4 & 51.3 & 26.5 & 36.7 \\
case5 & 34.8 & 18.1 & 25.8 \\
case6 & 39.8 & 24.0 & 27.5 \\
case7 & 31.7 & 12.0 & 27.2 \\
case8 & 24.5 & 22.4 & 8.2 \\
\hline Avg. & 32.5 & 20.3 & 20.5 \\
\hline
\end{tabular}

A part of this study was also supported by a JSPS Grantin-Aid for challenging Exploratory Research (grant number 18K19918). We thank Libby Cone, MD, MA, from Edanz Group Japan (www.edanzediting.com/ac) for editing a draft of this manuscript.

\section{REFERENCES}

[1] J. Tokuno, T. F. Chen-Yoshikawa, M. Nakao, T. Matsuda, H. Date, "Resection Process Map: A novel dynamic simulation system for pulmonary resection", The Journal of Thoracic and Cardiovascular Surgery, published online, 2019.

[2] M. Nakao, K. Minato, "Physics-based Interactive Volume Manipulation for Sharing Surgical Process", IEEE Trans. on Info. Tech. in Biomed., Vol. 14, No. 3, pp. 809-816, 2010.

[3] G. Gunay, L. M. Ha, T. V. Walsum, S. Klein, "Semi-automated registration of pre- and intra-operative liver CT for image-guided interventions", SPIE Medical Imaging, 97841N, 2016.

[4] P. Alvarez, M. Chabanas, S. Rouze, M. Castro, Y. Payan, J. L. Dillenseger, "Lung deformation between preoperative CT and intraoperative CBCT for thoracoscopic surgery: a case study", SPIE Medical Imaging, 105761D, 2018.

[5] J. Rühaak, T. Polzin, S. Heldmann, I. J. A. Simpson, H. Handels, J. Modersitzki, M. P. Heinrich, "Estimation of Large Motion in Lung CT by Integrating Regularized Keypoint Correspondences into Dense Deformable Registration", IEEE Transactions on Medical Imaging, Vol. 36, No. 8, pp. 1746-1757, 2017.

[6] K. Kobayashi, M. Nakao, J. Tokuno, T. F. Chen-Yoshikawa, T. Matsuda, "Analysis of deaeration deformation in ex vivo animal lung by Laplacian-based surface registration.”, In: 40th Annual international conference of the IEEE engineering in medicine and biology society (EMBC), 2018.

[7] M. Nakao, J. Tokuno, T. F. Chen-Yoshikawa, H. Date, T. Matsuda, "Surface Deformation Analysis of Collapsed Lungs using Modelbased Shape Matching", Int. J. Computer Assisted Radiology and Surgery, 14(10), pp. 1763-1774, 2019.

[8] A. Uneri, S. Nithiananthan, S. Schafer, Y. Otake, J. W. Stayman, G. Kleinszig, M.S. Sussman, J. L. Prince, J. H. Siewerdsen, "Deformable registration of the inflated and deflated lung in cone beam CT- guided thoracic surgery: Initial investigation of a combined moder and image - driven approach", Medical Physics, Vol. 40, Issue. 1, 017501, 2013.

[9] O. Oktay, L. Zhang, T. Mansi, P. Mountney, P. Mewes, S. Nicolau, L. Soler, C. Chefdhotel, "Biomechanically Driven Registration of Pre- to Intra- Operative 3D Images for Laparoscopic Surgery”, Medical Image Computing and Computer Assisted Intervention(MICCAI), vol. 8150, pp. 1-9, 2013.

[10] P. Alvarez, M. Chabanas, S. Rouze, M. Castro, Y. Payan, J. Dillenseger, "Lung deformation between preoperative CT and intraoperative CBCT for thoracoscopic surgery: a case study", SPIE medical imaging, Vol. 10576, pp. 1-7, 2018.

[11] A. Pitiot, E. Bardinet, P. M. Thompson,G. Malandain, "Piecewise affine registration of biological images for volume reconstruction", Medical Image Analysis, Vol. 10, Issue 3, pp. 465-483, 2006. 